JOURNAL OF THE

AMERICAN MATHEMATICAL SOCIETY

Volume 21, Number 4, October 2008, Pages 1001-1018

S 0894-0347(08)00608-5

Article electronically published on June 19, 2008

\title{
LOCALIZATION FOR QUANTUM GROUPS AT A ROOT OF UNITY
}

\author{
ERIK BACKELIN AND KOBI KREMNIZER
}

\section{INTRODUCTION}

Let $\mathbb{C}$ be the field of complex numbers and fix $q \in \mathbb{C}^{\star}$. Let $\mathfrak{g}$ be a semi-simple Lie algebra over $\mathbb{C}$ and let $G$ be the corresponding simply connected algebraic group. Let $U_{q}$ be the quantized enveloping algebra of $\mathfrak{g}$. Let $\mathcal{O}_{q}$ be the algebra of quantized functions on $G$. Let $\mathcal{O}_{q}(B)$ be the quotient Hopf algebra of $\mathcal{O}_{q}$ corresponding to a Borel subgroup $B$ of $G$.

In the paper BK] we defined categories of equivariant quantum $\mathcal{O}_{q}$-modules and $\mathcal{D}_{q}$-modules on the quantum flag variety of $G$. We proved that the BeilinsonBernstein localization theorem holds at a generic $q$. Namely, the global section functor gives an equivalence between categories of $U_{q}$-modules and $\mathcal{D}_{q}$-modules on the quantum flag variety. Thus one can translate questions about the representation theory of quantum groups to the study of the (noncommutative) geometry of the quantum flag variety. In this paper we prove that a derived version of this theorem holds at the root of unity case. Using this equivalence, we get that one can understand the representation theory of quantum groups at roots of unity through the (now-commutative) geometry of the Springer fibers.

We now recall the main results in $[\mathrm{BK}]$. The constructions given there are crucial for the present paper and a fairly detailed survey of the material there is given in the next section. We defined an equivariant sheaf of quasi-coherent modules over the quantum flag variety to be a left $\mathcal{O}_{q}$-module equipped with a right $\mathcal{O}_{q}(B)$-comodule structure satisfying certain compatibility conditions. Such objects form a category denoted $\mathcal{M}_{B_{q}}\left(G_{q}\right)$. It contains certain line bundles $\mathcal{O}_{q}(\lambda)$ for $\lambda$ in the weight lattice. We proved that $\mathcal{O}_{q}(\lambda)$ is ample for $\lambda>>0$ holds for every $q$. This implies that the category $\mathcal{M}_{B_{q}}\left(G_{q}\right)$ is a Proj-category in the sense of Serre.

We defined the category $\mathcal{D}_{B_{q}}^{\lambda}\left(G_{q}\right)$ of $\lambda$-twisted quantum $\mathcal{D}$-modules on the quantized flag variety (see Definition 3.11). An object $M \in \mathcal{D}_{B_{q}}^{\lambda}\left(G_{q}\right)$ is an object in $\mathcal{M}_{B_{q}}\left(G_{q}\right)$ equipped with an additional left $U_{q}$-action satisfying certain compatibility axioms (in particular, the $U_{q}(\mathfrak{b})$-action on $M$, that is, the restriction of the $U_{q}$-action, and the $\mathcal{O}_{q}(B)$-coaction "differ by $\lambda$ ").

Received by the editors November 1, 2006.

2000 Mathematics Subject Classification. Primary 14A22, 17B37, 58B32; Secondary $20 \mathrm{G} 42$.

Key words and phrases. Quantum groups, roots of unity, localization, derived equivalence, Calabi-Yau categories, noncommutative algebraic geometry.

The second author was supported in part by NSF grant DMS-0602007.

(C)2008 American Mathematical Society Reverts to public domain 28 years from publication 
The global section functor $\Gamma$ on $\mathcal{M}_{B_{q}}\left(G_{q}\right)$ (and on $\mathcal{D}_{B_{q}}^{\lambda}\left(G_{q}\right)$ ) is given by taking $\mathcal{O}_{q}(B)$-coinvariants. Let $M \in \mathcal{M}_{B_{q}}\left(G_{q}\right)$. Its sections over an open set (i.e., a localization $\mathcal{O}_{q f}$ of $\left.\mathcal{O}_{q}\right)$ are given by $\Gamma\left(\mathcal{O}_{q f}, M\right)=\Gamma\left(\mathcal{O}_{q f} \otimes_{\mathcal{O}_{q}} M\right)$.

The category $\mathcal{D}_{B_{q}}^{\lambda}\left(G_{q}\right)$ has a distinguished object $\mathcal{D}_{q}^{\lambda}$. As a vector space, $\mathcal{D}_{q}^{\lambda}=$ $\mathcal{O}_{q} \otimes M_{\lambda}$, where $M_{\lambda}$ is a Verma module. Here $\mathcal{D}_{q}^{\lambda}$ is the (quantum) equivariant counterpart of the usual sheaf of rings of $\lambda$-twisted differential operators on the flag variety of $G$. (The "set" $\mathcal{D}_{q}^{\lambda}$ is not a ring, but its "sections over open sets" are naturally rings.) We proved that for each $q$ except a finite set of roots of unity (depending on $\mathfrak{g}$ ), the global sections $\Gamma\left(\mathcal{D}_{q}^{\lambda}\right)$ is isomorphic to $U_{q}^{\lambda}:=U_{q}^{f i n} / J_{\lambda}$, where $J_{\lambda}$ is the annihilator of $M_{\lambda}$ and $U_{q}^{f i n}$ is the maximal subalgebra of $U_{q}$ on which the adjoint action of $U_{q}$ is locally finite.

The main result in $[\mathrm{BK}]$ stated that the global section functor gives an equivalence of categories between $\mathcal{D}_{B_{q}}^{\lambda}\left(G_{q}\right)$ and modules over $U_{q}^{\lambda}$ in the case when $q$ is not a root of unity and $\lambda$ is regular dominant. This is a quantum version of Beilinson-Bernstein's, BB, localization theorem.

In the present paper we study the root of unity case; $q$ is a primitive $l$ 'th root of unity. The situation becomes different and very interesting: It turns out that $\mathcal{D}_{q}^{\lambda}$ naturally forms a sheaf of algebras over the classical (nonquantum) complex variety $T^{\star} X^{\lambda}:=\left(G \times N^{\lambda^{2 l}}\right) / B$, where $N^{\lambda^{2 l}}$ is the $B$-invariant subvariety $\left\{\lambda^{2 l}\right\} \times N$ of $B$ (see section 3.1). If $\lambda$ is integral, $N^{\lambda^{2 l}}=N$ and so $T^{\star} X^{\lambda}=T^{\star} X$ is the cotangent bundle of the flag variety $X$ of $G$ in this case. Hence, we refer to $T^{\star} X^{\lambda}$ as a twisted cotangent bundle.

A key observation is that $\mathcal{D}_{q}^{\lambda}$ is an Azumaya algebra over a dense subset of $T^{\star} X^{\lambda}$ (Proposition 3.6) and that this Azumaya algebra splits over formal neighborhoods of generalized Springer fibers (Proposition 3.23). We then show that $\mathcal{D}_{q}^{\lambda}$ has no higher self-extensions, i.e., that $R \Gamma^{>0}\left(\mathcal{D}_{q}^{\lambda}\right)=0$ (Proposition 3.25) . This fact, together with the result $\Gamma\left(\mathcal{D}_{q}^{\lambda}\right)=U_{q}^{\lambda}$ and the Azumaya property implies a derived version of the localization theorem: The functor $\Gamma$ induces an equivalence between bounded derived categories $D^{b}\left(\mathcal{D}_{B_{q}}^{\lambda}\left(G_{q}\right)\right)$ and $D^{b}\left(U_{q}^{\lambda}-\bmod \right)$ (restricted to the dense subsets).

Using the Azumaya splitting, we prove that the subcategory of $\mathcal{D}_{B_{q}}^{\lambda}\left(G_{q}\right)$ whose objects are supported on (a formal neighbourhood of a generalized) Springer fiber is isomorphic to the category of $\mathcal{O}$-modules over the twisted cotangent bundles $T^{\star} X^{\lambda}$ supported on the same fiber (Corollary 3.24).

Combining these results, we get an equivalence between $D^{b}\left(U_{q}^{\lambda}-\bmod \right)$ and the derived category of $\mathcal{O}$-modules on $T^{\star} X^{\lambda}$ whose cohomologies are supported on certain Springer fibers.

An application of our theory is for instance the computation of the number of simple $\mathfrak{u}_{q}$-modules, because this number can be interpreted as the rank of the $K$ group of the category of $\mathcal{O}$-modules on $T^{\star} X^{\lambda}$ supported on the trivial Springer fiber. Of course, such a formula follows also from the fact that this is a highest weight category. Our method gives the possibility to extend this to nontrivial central characters as well. In a future paper we will use this to prove a conjecture of De Concini, Kac and Procesi regarding dimensions of irreducible modules.

In BMR analogous results were established for a Lie algebra $\mathfrak{g}_{p}$ in characteristic $p$. In fact most of our methods of proofs are borrowed from that paper. They showed analogous results for $U\left(\mathfrak{g}_{p}\right)$-modules, (crystalline) $\mathcal{D}$-modules 
on the flag variety over the finite field $X_{\mathbb{F}_{p}}$ and certain twisted cotangent bundles of $X$ over $\overline{\mathbb{F}}_{p}$.

Combining their results with ours, we see that the representation theory of $U_{q}$ (when $q$ is a $p$ 'th root of unity) is related to the representation theory of $U\left(\mathfrak{g}_{p}\right)$ via cotangent bundles of $X$ in char 0 and $\operatorname{char} p$, respectively. We know furthermore that baby Verma modules go to skyscraper sheaves in both cases and BMR] showed that the $K$-group of the category of quasi-coherent sheaves on the cotangent bundle is torsion-free and its rank does not depend on the characteristic of the field. Right now we are investigating what can be deduced about the representation theory of $\mathfrak{g}_{p}$, e.g., character formulas, from the representation theory of $U_{q}$ with these methods.

\section{Generalities}

2.1. Quantum groups. See Chari and Pressley $\mathrm{CP}$ for details about the topics in this section.

2.1.1. Conventions. Let $\mathbb{C}$ be the field of complex numbers and fix $q \in \mathbb{C}^{\star}$.

We always assume that if $q$ is a root of unity, it is primitive of odd order and in case $G$ has a component of type $G_{2}$, the order is also prime to 3. Let $\mathcal{A}$ be the local ring $\mathbb{Z}[\nu]_{\mathfrak{m}}$, where $\mathfrak{m}$ is the maximal ideal in $\mathbb{Z}[\nu]$ generated by $\nu-1$ and a fixed odd prime $p$.

2.1.2. Root data. Let $\mathfrak{g}$ be a semi-simple Lie algebra and let $\mathfrak{h} \subset \mathfrak{b}$ be a Cartan subalgebra contained in a Borel subalgebra of $\mathfrak{g}$. Let $R$ be the root system, $\Delta \subset$ $R_{+} \subset R$ a basis and the positive roots. Let $P \subset \mathfrak{h}^{\star}$ be the weight lattice and $P_{+}$ the positive weights; the $i$ 'th fundamental weight is denoted by $\omega_{i}$ and $\rho$ denotes the half sum of the positive roots. Let $Q \subset P$ be the root lattice and $Q_{+} \subset Q$ those elements which have nonnegative coefficients with respect to the basis of simple roots. Let $\mathcal{W}$ be the Weyl group of $\mathfrak{g}$. We let $\langle$,$\rangle denote a \mathcal{W}$-invariant bilinear form on $\mathfrak{h}^{\star}$ normalized by $\langle\gamma, \gamma\rangle=2$ for each short root $\gamma$.

Let $T_{P}=\operatorname{Hom}_{\text {groups }}\left(P, \mathbb{C}^{\star}\right)$ be the character group of $P$ with values in $\mathbb{C}$ (we use additive notation for this group). If $\mu \in P$, then $\langle\mu, P\rangle \subset \mathbb{Z}$ and hence we can define $q^{\mu} \in T_{P}$ by the formula $q^{\mu}(\gamma)=q^{\langle\mu, \gamma\rangle}$, for $\gamma \in P$. If $\mu \in P, \lambda \in T_{P}$, we write $\mu+\lambda=q^{\mu}+\lambda$. Note that the Weyl group naturally acts on $T_{P}$.

2.1.3. Quantized enveloping algebra $U_{q}$ and quantized algebra of functions $\mathcal{O}_{q}$. Let $U_{q}$ be the simply connected quantized enveloping algebra of $\mathfrak{g}$ over $\mathbb{C}$. Recall that $U_{q}$ has algebra generators $E_{\alpha}, F_{\alpha}, K_{\mu}, \alpha, \beta$ are simple roots, $\mu \in P$ subject to the relations

$$
\begin{gathered}
K_{\lambda} K_{\mu}=K_{\lambda+\mu}, \quad K_{0}=1, \\
K_{\mu} E_{\alpha} K_{-\mu}=q^{\langle\mu, \alpha\rangle} E_{\alpha}, \quad K_{\mu} F_{\alpha} K_{-\mu}=q^{-\langle\mu, \alpha\rangle} F_{\alpha}, \\
{\left[E_{\alpha}, F_{\beta}\right]=\delta_{\alpha, \beta} \frac{K_{\alpha}-K_{-\alpha}}{q_{\alpha}-q_{\alpha}^{-1}}}
\end{gathered}
$$

and certain Serre relations that we do not recall here. Here

$$
\left.q_{\alpha}=q^{d_{\alpha}}, \quad d_{\alpha}=\angle \alpha, \alpha\right\rangle / 2 .
$$

(We have assumed that $q_{\alpha}^{2} \neq 1$.) 
Let $G$ be the simply connected algebraic group with Lie algebra $\mathfrak{g}, B$ a Borel subgroup of $G$ and $N \subset B$ its unipotent radical. Let $\mathfrak{b}=\operatorname{Lie} B$ and $\mathfrak{n}=\operatorname{Lie} N$ and denote by $U_{q}(\mathfrak{b})$ and $U_{q}(\mathfrak{n})$ the corresponding subalgebras of $U_{q}$. Then $U_{q}(\mathfrak{b})$ is a Hopf algebra, while $U_{q}(\mathfrak{n})$ is only an algebra. Let $\mathcal{O}_{q}=\mathcal{O}_{q}(G)$ be the algebra of matrix coefficients of finite dimensional type- 1 representations of $U_{q}$. There is a natural pairing $():, U_{q} \otimes \mathcal{O}_{q} \rightarrow \mathbb{C}$. This gives a $U_{q}$-bimodule structure on $\mathcal{O}_{q}$ as follows:

$$
u a=a_{1}\left(u, a_{2}\right), \quad a u=\left(u, a_{1}\right) a_{2}, \quad u \in U_{q}, \quad a \in \mathcal{O}_{q} .
$$

Then $\mathcal{O}_{q}$ is the (restricted) dual of $U_{q}$ with respect to this pairing. We let $\mathcal{O}_{q}(B)$ and $\mathcal{O}_{q}(N)$ be the quotient algebras of $\mathcal{O}_{q}$ corresponding to the subalgebras $U_{q}(\mathfrak{b})$ and $U_{q}(\mathfrak{n})$ of $U_{q}$, respectively, by means of this duality. Then $\mathcal{O}_{q}(B)$ is a Hopf algebra and $\mathcal{O}_{q}(N)$ is only an algebra.

There is a braid group action on $U_{q}$. For each $w \in \mathcal{W}$, we get an automorphism $T_{w}$ of $U_{q}$.

2.1.4. Integral versions of $U_{q}$. Let $U_{\mathcal{A}}^{\text {res }}$ be the Lusztig integral form of $U_{q}$, the $\mathcal{A}$ algebra in $U_{q}$ generated by divided powers $E_{\alpha}^{(n)}=E_{\alpha}^{n} /[n]_{d_{\alpha}} !, F_{\alpha}^{(n)}=F_{\alpha}^{n} /[n]_{d_{\alpha}}$ !, $\alpha$ a simple root, $n \geq 1$ (where $[m]_{d}=\frac{\prod_{s=1}^{m} q^{d \cdot s}-q^{-d \cdot s}}{q^{d}-q^{-d}}$ ) and the $K_{\mu}$ 's, $\mu \in P$. There is also the De Concini-Kac integral form $U_{\mathcal{A}}$, which is generated over $\mathcal{A}$ by the $E_{\alpha}, F_{\alpha}$ and $K_{\mu}$ 's. The subalgebra $U_{\mathcal{A}}$ is preserved by the adjoint action of $U_{\mathcal{A}}^{r e s}: \operatorname{ad}_{U_{\mathcal{A}}^{\text {res }}}\left(U_{\mathcal{A}}\right) \subset U_{\mathcal{A}}$. The operators $T_{w}$ from section 2.1.2 preserve the integral versions.

$\mathcal{O}_{\mathcal{A}}$ is defined to be the dual of $U_{\mathcal{A}}^{\text {res }}$. This is an $\mathcal{A}$-sub-Hopf algebra of $\mathcal{O}_{q}$.

2.1.5. Finite part of $U_{q}$. The algebra $U_{q}$ acts on itself by the adjoint action ad : $U_{q} \rightarrow U_{q}$ where $\operatorname{ad}(u)(v)=u_{1} v S\left(u_{2}\right)$. Let $U_{q}^{f i n}$ be the finite part of $U_{q}$ with respect to this action:

$$
U_{q}^{f i n}=\left\{v \in U_{q} ; \operatorname{dim} \operatorname{ad}\left(U_{q}\right)(v)<\infty\right\} .
$$

This is a subalgebra. (See [JL.)

We can also give an integral version of the finite part as the finite part of the action of $U_{\mathcal{A}}^{r e s}$ on $U_{\mathcal{A}}$. Thus by specializing we get a subalgebra of $U_{q}$ for every $q$. Of course, when specialized to generic $q$, this coincides with the previous definition.

2.1.6. Specializations and Frobenius maps. For any ring map $\phi: \mathcal{A} \rightarrow R$ we put $U_{R}=U_{\mathcal{A}} \otimes_{\mathcal{A}} R$ and $U_{R}^{\text {res }}=U_{\mathcal{A}}^{\text {res }} \otimes_{\mathcal{A}} R$. If $R=\mathbb{C}$ and $\phi(\nu)=q$, there are three different cases: $q$ is a root of unity, $q=1$ and $q$ is generic. Then $U_{R}=U_{q}$.

There is also a ring map $\mathcal{A} \rightarrow \mathbb{F}_{p}$, sending $\nu \rightarrow 1$. Then $U_{\mathbb{F}_{p}} /(K-1)=U\left(\mathfrak{g}_{p}\right)$, the enveloping algebra of the Lie algebra $\mathfrak{g}_{p}$ in characteristic $p$.

For any $U_{\mathcal{A}}$-module (resp. $U_{\mathcal{A}}^{r e s}$-module) $M_{\mathcal{A}}$ we put $M_{R}=M_{\mathcal{A}} \otimes_{\mathcal{A}} R$. This is an $U_{R}$-module (resp. $U_{R}^{\text {res }}$-module). When $R=\mathbb{C}$, we simply write $M=M_{\mathbb{C}}$.

When $q$ is a root of unity, we have the Frobenius map: $U_{q}^{\text {res }} \rightarrow U(\mathfrak{g})$. Its algebra kernel is denoted by $\mathfrak{u}_{q}$. We also have the Frobenius map $U_{q}^{\text {res }}(\mathfrak{b}) \rightarrow U(\mathfrak{b})$, with algebra kernel $b_{q}$. These maps induce dual maps $\mathcal{O}=\mathcal{O}(G) \hookrightarrow \mathcal{O}_{q}$ and $\mathcal{O}(B) \hookrightarrow \mathcal{O}_{q}(B)$. It is important to note that the Frobenius map $U_{q}^{r e s}(\mathfrak{b}) \rightarrow U(\mathfrak{b})$ has a splitting. From this splitting we can deduce that the functor of $b_{q}$-invariants on the category of $U_{q}^{\text {res }}(\mathfrak{b})$ is exact as follows: the splitting implies that $U_{q}^{\text {res }}(\mathfrak{b})$ is a semi-direct product of $b_{q}$ and $U(\mathfrak{b})$. Hence $b_{q}$ is exact in $U_{q}^{r e s}(\mathfrak{b})$ (the induction 
functor is exact). Note that we are working with algebraic induction so we should write everything in terms of the dual Hopf algebras. So we get

$$
\begin{aligned}
& H^{*}\left(b_{q}, \operatorname{Res}_{b_{q}}^{B^{q}}(M)\right)=H^{*}\left(B_{q}, \operatorname{Ind}_{b_{q}}^{B^{q}} \operatorname{Res}_{b_{q}}^{B^{q}}(M)\right) \\
& \quad=H^{*}\left(B_{q}, O(B) \otimes M^{\text {triv }}\right)=H^{*}\left(B_{q}, \operatorname{Ind}_{b_{q}}^{B^{q}}(\mathbb{C})\right) \otimes M=H^{*}\left(b_{q}, \mathbb{C}\right) \otimes M .
\end{aligned}
$$

Here $H^{*}\left(B_{q}, ?\right)$ is cohomology w.r.t. the big quantum Borel. Note that the cohomology of $b_{q}$ is zero in odd degrees. Hence when we apply the functor of invariants to an exact sequence of integrable $U_{q}^{\text {res }}(\mathfrak{b})$-modules, we get an exact sequence. Note that we have proven a stronger statement, we only need to assume that the first object in the sequence is a $B_{q}$-module.

For each $q$ there exists a map $U_{q} \rightarrow U_{q}^{\text {res }}$ whose image is $\mathfrak{u}_{q}$ and whose algebra kernel is $\mathcal{Z}^{(l)}$ (see section 2.1.7 below for the definition of $\mathcal{Z}^{(l)}$ ).

2.1.7. Verma modules. For each $\lambda \in T_{P}$ there is the one dimensional $U_{q}(\mathfrak{b})$-module $\mathbb{C}_{\lambda}$ which is given by extending $\lambda$ to act by zero on the $E_{\alpha}$ 's. The Verma module $M_{\lambda}$ is the $U_{q}$-module induced from $\mathbb{C}_{\lambda}$. If $\mu \in P$, we write $M_{\mu}=M_{q^{\mu}}$. An important point for us is that $M_{\lambda}$ carries an $U_{q}^{\text {res }}(\mathfrak{b})$-module structure defined as follows: $U_{q}^{\text {res }}(\mathfrak{b})$ acts on $U_{q}$ by restricting the adjoint action of $U_{q}^{\text {res }}$ on $U_{q}$. This induces a $U_{q}(\mathfrak{b})$-action on the quotient $M_{\lambda}$ of $U_{q}$. Since this action is locally finite, it corresponds to an $\mathcal{O}_{q}(B)$-comodule action on $M_{\lambda}$. Note!! As a $U_{q}^{\text {res }}(\mathfrak{b})$-module $M_{\lambda}$ has trivial highest weight. (In case $q$ is generic, $U_{q}^{r e s}(\mathfrak{b})=U_{q}(\mathfrak{b})$ and then the $U_{q}^{\text {res }}(\mathfrak{b})$-action on $M_{\lambda}$ described above is the same as the $U(\mathfrak{b})$-action on $M_{\lambda} \otimes \mathbb{C}_{-\lambda}$.)

The Verma module $M_{\lambda}$ has an integral version $M_{\lambda, \mathcal{A}}$.

2.1.8. Centers of $U_{q}$ and definition of $\widetilde{U}_{q}$. Let $\mathcal{Z}$ denote the center of $U_{q}$. When $q$ is a $p^{\prime}$ th root of unity, $\mathcal{Z}$ contains the Harish-Chandra center $\mathcal{Z}^{H C}$ and the l'th center $\mathcal{Z}^{(l)}$ which is generated by the $E_{\alpha}^{l}, F_{\alpha}^{l}, K_{\mu}^{l}$ and $K_{\mu}^{-l}$ 's. In fact, $\mathcal{Z}=$ $\mathcal{Z}^{(l)} \otimes_{\mathcal{Z}^{(l)} \cap \mathcal{Z}^{H C}} \mathcal{Z}^{H C}$. There is the Harish-Chandra homomorphism $\mathcal{Z}^{H C} \rightarrow \mathcal{O}\left(T_{P}\right)$ that maps isomorphically to the $W$-invariant even part of $\mathcal{O}\left(T_{P}\right)$. We define $\widetilde{U}_{q}=$ $U_{q} \otimes_{\mathcal{Z}^{H C}} \mathcal{O}\left(T_{P}\right)$.

2.1.9. Some conventions. We shall frequently refer to a right (resp. left) $\mathcal{O}_{q}$-comodule as a left (resp. right) $G_{q}$-module, etc. If we have two right $\mathcal{O}_{q}$-comodules $V$ and $W$, then $V \otimes W$ carries the structure of a right $\mathcal{O}_{q}$-comodule via the formula

$$
\delta(v \otimes w)=v_{1} \otimes w_{1} \otimes v_{2} w_{2} .
$$

We shall refer to this action as the tensor or diagonal action. A similar formula exist for left comodules.

2.2. Quantum flag variety. Here we recall the definition and basic properties of the quantum flag variety from $\mathrm{BK}$.

2.2.1. Category $\mathcal{M}_{B_{q}}\left(G_{q}\right)$. The composition

$$
\mathcal{O}_{q} \rightarrow \mathcal{O}_{q} \otimes \mathcal{O}_{q} \rightarrow \mathcal{O}_{q} \otimes \mathcal{O}_{q}(B)
$$

defines a right $\mathcal{O}_{q}(B)$-comodule structure on $\mathcal{O}_{q}$. A $B_{q}$-equivariant sheave on $G_{q}$ is a triple $(F, \alpha, \beta)$ where $F$ is a vector space, $\alpha: \mathcal{O}_{q} \otimes F \rightarrow F$ a left $\mathcal{O}_{q}$-module action and $\beta: F \rightarrow F \otimes \mathcal{O}_{q}(B)$ a right $\mathcal{O}_{q}(B)$-comodule action such that $\alpha$ is a right comodule map, where we consider the tensor comodule structure on $\mathcal{O}_{q}(G) \otimes F$. 
Definition 2.1. We denote $\mathcal{M}_{B_{q}}\left(G_{q}\right)$ to be the category of $B_{q}$-equivariant sheaves on $G_{q}$. Morphisms in $\mathcal{M}_{B_{q}}\left(G_{q}\right)$ are those compatible with all structures.

If $q=1$, the category $\mathcal{M}_{B}(G)$ is equivalent to the category $\mathcal{M}(G / B)$ of quasicoherent sheaves on $G / B$.

Definition 2.2. We define the induction functor Ind : $B_{q}-\bmod$ to $\mathcal{M}_{B_{q}}\left(G_{q}\right)$, Ind $V=\mathcal{O}_{q} \otimes V$ with the tensor $B_{q}$-action and the $\mathcal{O}_{q}$-action on the first factor. For $\lambda \in P$ we define a line bundle $\mathcal{O}_{q}(\lambda)=\operatorname{Ind} \mathbb{C}_{-\lambda}$.

Definition 2.3. The global section functor $\Gamma: \mathcal{M}_{B_{q}}\left(G_{q}\right) \rightarrow \mathbb{C}-\bmod$ is defined by

$$
\Gamma(M)=\operatorname{Hom}_{\mathcal{M}_{B_{q}}\left(G_{q}\right)}\left(\mathcal{O}_{q}, M\right)=\left\{m \in M ; \Delta_{B}(m)=m \otimes 1\right\} .
$$

This is the set of $B_{q}$-invariants in $M$.

The category $\mathcal{M}_{B_{q}}\left(G_{q}\right)$ has enough injectives, so derived functors $R \Gamma$ are welldefined. We showed that $R^{i} \Gamma(\operatorname{Ind} V)=H^{i}\left(G_{q} / B_{q}, V\right)$, where $H^{i}\left(G_{q} / B_{q},\right)$ is the $i$ 'th derived functor of the functor $V \rightarrow \Gamma($ Ind $V)$ from $B_{q}-\bmod$ to $\mathbb{C}-\bmod$.

We proved a quantum version of Serre's basic theorem on projective schemes: Each $M \in \mathcal{M}_{B_{q}}\left(G_{q}\right)$ is a quotient of a direct sum of $\mathcal{O}_{q}(\lambda)$ 's and each surjection $M \rightarrow M^{\prime}$ of noetherian objects in $\mathcal{M}_{B_{q}}\left(G_{q}\right)$ induces a surjection $\Gamma(M(\lambda)) \rightarrow$ $\Gamma\left(M^{\prime}(\lambda)\right)$ for $\lambda>>0$.

Here the notation $\lambda>>0$ means that $\left\langle\lambda, \alpha^{\wedge}\right\rangle$ is a sufficiently large integer for each simple root $\alpha$ and $M(\lambda)=M \otimes \mathbb{C}_{-\lambda}$ is the $\lambda$-twist of $M$.

Let $V \in G_{q}-\bmod$. Denote by $V \mid B_{q}$ the restriction of $V$ to $B_{q}$ and by $V^{\text {triv }}$ the trivial $B_{q}$-module whose underlying space is $V$. We showed that $\operatorname{Ind} V \mid B_{q}$ and Ind $V^{\text {triv }}$ are isomorphic in $\mathcal{M}_{B_{q}}\left(G_{q}\right)$. In particular

$$
\Gamma\left(\text { Ind } V \mid B_{q}\right)=V\left|B_{q} \otimes \Gamma\left(\mathcal{O}_{q}\right)=V\right| B_{q}, \quad \text { for } V \in G_{q}-\bmod .
$$

2.2.2. $\mathcal{M}_{B_{q}}\left(G_{q}\right)$ at a root of unity. In case $q$ is a root of unity, we have the following Frobenius morphism:

$$
F r_{*}: \mathcal{M}_{B_{q}}\left(G_{q}\right) \rightarrow \mathcal{M}(G / B)
$$

defined as

$$
N \mapsto N^{b_{q}} .
$$

Using the description of $\mathcal{M}_{B_{q}}\left(G_{q}\right)$ as $\operatorname{Proj}\left(A_{q}\right)$ where $A_{q}=\bigoplus V_{q, \lambda}$ BK and similarly $\mathcal{M}(G / B)=\operatorname{Proj}(A)$ where $A=\bigoplus V_{\lambda}$, we see that $F r_{*}$ is induced from the quantum Frobenius map $A \hookrightarrow A_{q}$. It follows that

Proposition 2.4. $F r_{*}$ is exact and faithful.

This functor has a left adjoint.

\section{3. $\mathcal{D}_{q}^{\lambda}$-MOdules AT A RoOT OF Unity}

3.1. First construction. From now on $q$ is an $l$ 'th root of unity (recall the restrictions of 2.1.1).

In this section we shall give a representation theoretic construction of the sheaf of quantum differential operators. This will turn out to be a sheaf of algebras over the Springer resolution - the sheaf of endomorphisms of the (nonexistent) universal baby Verma module. 
Recall the Frobenius map $O \hookrightarrow O_{q}$ and the fact that $O_{q}^{\mathfrak{u}_{q}}=O$. This allows us to define the functor of (finite) induction

$$
\begin{aligned}
\text { Ind }: \mathfrak{u}_{q}-\bmod \rightarrow \mathcal{M}_{G_{q}}(G), \\
N \mapsto\left(O_{q} \otimes N\right)^{\mathfrak{u}_{q}} .
\end{aligned}
$$

Here $\mathcal{M}_{G_{q}}(G)$ is the category of $G_{q}$-equivariant $O$-modules, that is, an $O$-module which is also an $O_{q}$-comodule and such that the $O$-module structure map is a map of $O_{q}$-comodules.

We have the following important proposition $[\mathrm{AG}$ :

Proposition 3.1. Ind $: \mathfrak{u}_{q}-\bmod \rightarrow \mathcal{M}_{G_{q}}(G)$ is an equivalence of categories.

Notice that both categories are tensor categories (in $\mathcal{M}_{G_{q}}(G)$ it is tensoring over $O$ ) and that Ind is a tensor functor. This will be used later. Note also that $\mathcal{M}_{G_{q}}(G)$ has an obvious action by $G$, that is, for any $g \in G$ we have a functor $F_{g}: \mathcal{M}_{G_{q}}(G) \rightarrow \mathcal{M}_{G_{q}}(G)$ and natural transformations $\alpha_{g, h}: F_{g} \circ F_{h} \Rightarrow F_{g h}$ satisfying a certain cocycle condition. Moreover starting from any $\mathfrak{u}_{q}$-module, we can form a family of such modules indexed by $G$, more precisely an $O(G)$-module in the category of $\mathfrak{u}_{q}$-modules. We will use this to construct the universal family of endomorphisms of baby Verma modules.

Remark 3.2. The article CKP defined an action of an infinite dimensional group $\mathfrak{G}$ on $U_{q}$ preserving the $l$-center (and the augmentation ideal of the $l$-center) and thus acting also on $\mathfrak{u}_{q}$. This is defined by observing that the derivation defined by commuting with the divided powers $E^{(l)}, F^{(l)}$ actually preserves the algebra generated by the nondivided powers. These derivations are then exponentiated to get automorphisms of $U_{q}$ at a root of unity. The group they generate is infinite dimensional as the action is not locally finite. This action also induces an action on $\mathfrak{u}_{q}$, but here the group is finite dimensional $\mathfrak{G}_{0}$. We thus have another group action on the category. Note that the action of $G$ on the category gives us an infinitesimal action of the Lie algebra $\mathfrak{g}$ on the category, which as weak action is the same as a map of $\mathfrak{g}$ to outer derivations. But for $\mathfrak{G}_{0}$ the Lie algebra action is given by the derivation defined by commuting with a divided power and for $G$ it is given by the adjoint action and both have the same image inside $\operatorname{Ext}^{1}\left(\mathfrak{u}_{q}, \mathfrak{u}_{q}\right)$. Both actions are also faithful, so as weak actions they are the same.

Recall that $M_{\lambda}=U_{q} / J_{\lambda}$; put

$$
\begin{gathered}
I_{\lambda}=\mathcal{Z}^{(l)} \cap J_{\lambda}, \\
\mathcal{Z}_{-, \lambda}^{(l)}=\mathcal{Z}^{(l)} / I_{\lambda},
\end{gathered}
$$

so that $\mathcal{Z}_{-, \lambda}^{(l)} \subset M_{\lambda}$.

Then $\mathcal{Z}_{-, \lambda}^{(l)}$ is a $U_{q}^{\text {res }}(\mathfrak{b})$-module algebra and $M_{\lambda}$ is a $U_{q}^{\text {res }}(\mathfrak{b})$-module for this algebra (see section 2.1.7).

In fact, $\mathcal{O} \otimes \mathcal{Z}_{-, \lambda}^{(l)}$ is a $B$-equivariant algebra: Recall the Frobenius map $\mathrm{Fr}$ : $U_{q}^{r e s}(\mathfrak{b}) \rightarrow U(\mathfrak{b})$ and denote the algebra kernel of $F r$ by $b_{q}$.

Definition 3.3. i) Consider $\mathcal{O}(B)$ as a $B$-module under the adjoint action. For each $t \in T$ we have the $B$-subvariety $N^{t}=\{t\} \times N$ of $B . \mathcal{O}\left(N^{t}\right)$ is isomorphic to $\mathcal{O}(N)$ as an algebra, but not as a $B$-module, unless $t=1$. 
ii) Think of $T$ as characters on $2 l \cdot P$ and of $T_{P}$ as characters on $P$. Lattice inclusion induces a natural map ()$^{2 l}: T_{P} \rightarrow T$.

Note that for $\lambda$ integral, $\lambda^{2 l}=1$.

We now have

Lemma 3.4. $b_{q}$ acts trivially on $\mathcal{Z}_{-, \lambda}^{(l)}$; this module is a pullback by the Frobenius of the B-module $\mathcal{O}\left(N^{\lambda^{2 l}}\right)$.

Proof of Lemma 3.4. This will follow from Lemma 3.18

$b_{q}$ also acts trivially on $\mathcal{O}$, so $\mathcal{O} \otimes \mathcal{Z}_{-, \lambda}^{(l)}$ is a $B$-module. Hence, we have the category $\mathcal{M}_{B}\left(\mathcal{O} \otimes \mathcal{Z}_{-, \lambda}^{(l)}\right)$ of $B$-equivariant $\mathcal{O} \otimes \mathcal{Z}_{-, \lambda}^{(l)}$-modules; from Lemma 3.4 we conclude that

$$
\mathcal{M}_{B}\left(\mathcal{O} \otimes \mathcal{Z}_{-, \lambda}^{(l)}\right) \cong q \operatorname{coh}\left(\mathcal{O}_{\left(G \times N^{\lambda^{2 l}}\right) / B}\right) .
$$

Here the $B$-action on $G \times N^{\lambda}$ is given by $b \cdot(g, x)=(b g, b \cdot x)$. Similarly we can define the category ${ }_{G_{q}} \mathcal{M}_{B}\left(\mathcal{O} \otimes \mathcal{Z}_{-, \lambda}^{(l)}\right)$ of $G_{q^{-}}$equivariant objects in $\mathcal{M}_{B}\left(\mathcal{O} \otimes \mathcal{Z}_{-, \lambda}^{(l)}\right)$.

We shall denote

$$
G \times N^{\lambda^{2 l}} / B=T^{\star} X^{\lambda} .
$$

Note that for integral $\lambda$ this is the cotangent bundle to the flag variety, also known as the Springer resolution. For nonintegral $\lambda$ this is a twisted cotangent bundle.

For any $\tau \in \max \operatorname{spec}\left(\mathcal{Z}_{-, \lambda}^{(l)}\right)$ we have the central reduction $M_{\lambda, \tau}$ (a baby Verma module). Only for trivial $\tau$ (corresponding to the augmentation ideal) we get a $\mathfrak{u}_{q^{-}}$ module. But for any $\tau, \operatorname{End}\left(M_{\lambda, \tau}\right)=M_{\lambda, \tau} \otimes M_{\lambda, \tau}^{*}$ is a $\mathfrak{u}_{q}$-module since its $l$-central character is trivial, and likewise $\operatorname{End}_{\mathcal{Z}_{-, \lambda}^{(l)}}\left(M_{\lambda}\right)$ is a $\mathfrak{u}_{q}$-module. Hence we can define

Definition 3.5. $D=\left(\operatorname{Ind}\left(\operatorname{End}_{\mathcal{Z}_{-, \lambda}^{(l)}}\left(M_{\lambda+2 \rho}\right)\right)\right)$.

(The shift by $2 \rho$ will become clear later.) Since $\operatorname{End}_{\mathcal{Z}_{-, \lambda}^{(l)}}\left(M_{\lambda}\right)$ is a $\mathcal{Z}_{-, \lambda^{(}}^{(l)}$-module and also a $B_{q}$-module $\left(O_{q}(B)\right.$-comodule) in a compatible way we get that $D$ actually lives in ${ }_{G_{q}} \mathcal{M}_{B}\left(\mathcal{O} \otimes \mathcal{Z}_{-, \lambda}^{(l)}\right)$. Since Ind is a tensor functor and $\operatorname{End}_{\mathcal{Z}_{-, \lambda}^{(l)}}\left(M_{\lambda}\right)$ is a $\mathfrak{u}_{q}$ algebra, we get that $D$ is a sheaf of algebras over the (twisted) cotangent bundle.

By construction we know that the algebras sitting over the fiber over $B$ are matrix algebras (endomorphisms of baby Verma modules); hence we get

Proposition 3.6. Over a dense subset of $T^{\star} X^{\lambda}, D$ is an Azumaya algebra.

Note that by the construction of the sheaf of algebras $D$, its fibers over the zero section of $T^{\star} X^{\lambda}$ are endomorphisms of baby Verma modules. Hence we see that over the zero section $D$ is the endomorphism sheaf of the vector bundle whose fibers are baby Verma modules. This implies that the Azumaya locus contains the zero section and in fact the algebra is split over the zero section. It will also follow from Proposition 3.23 that the Azumaya locus contains the preimage of the big cell under the Springer map.

Remark 3.7. Note that all our constructions can also be defined over a formal neighbourhood of a prime $p$ that is over a $p$-adic field and that when specialized to $\mathbf{F}_{p}$, they give the usual characteristic $p$ crystalline differential opeartors which are 
Azumaya and thus we would get that over the $p$-adic field our algebra is Azumaya as well. This will not be used in this paper since we will look at complex representations, but in a subsequent paper we will use this to construct $t$-structures in zero characteristic relating to the ones constructed by Bezrukavnikov, Mirkovic and Rumynin BMR.

Remark 3.8. For any rigid braided tensor category one can define the notion of an Azumaya algebra. In the category of $\mathfrak{u}_{q}$-modules, $\operatorname{End}_{\mathcal{Z}_{-, \lambda}^{(l)}}\left(M_{\lambda}\right)$ is an Azumaya algebra. Hence, using the equivalence Proposition 3.1 we get that $D$ is an Azumaya algebra over $T^{\star} X^{\lambda}$, not with respect to the usual braiding (flip), but with respect to the braiding induced from $U_{q}^{\text {res }}$.

3.2. Second construction - the ring $\mathcal{D}_{G_{q}}$ and the category of $\mathcal{D}^{\lambda}$-modules. We need the following important

Remark 3.9. All objects described in the preceding sections are defined over $\mathcal{A}$. For any specialization $\mathcal{A} \rightarrow R$ and any object $O b j$ we denote by $O b j_{R}$ its $R$-form. For the functors, we do not use any subscripts; so, for instance, there is the functor Ind $: B_{q, R}-\bmod \rightarrow \mathcal{M}_{B_{q}}\left(G_{q}\right)_{R}$.

Recall the $U_{q}$-bimodule structure on $\mathcal{O}_{q}$ given by (2.4). Now, as we have two versions of the quantum group, we pick the following definition of the ring of differential operators on the group (the crystalline version).

Definition 3.10. We define the ring of quantum differential operators on $G_{q}$ to be the smash product algebra $\mathcal{D}_{G_{q}}:=\mathcal{O}_{q} \star U_{q}$. So $\mathcal{D}_{G_{q}}=\mathcal{O}_{q} \otimes U_{q}$ as a vector space and multiplication is given by

$$
a \otimes u \cdot b \otimes v=a u_{1}(b) \otimes u_{2} v .
$$

We consider now the ring $\mathcal{D}_{G_{q}}$ as a left $U_{q}^{\text {res }}$-module, via the left $U_{q}^{\text {res }}$-action on $\mathcal{O}_{q}$ in (2.4) and the left adjoint action of $U_{q}^{\text {res }}$ on $U_{q}$; this way $\mathcal{D}_{G_{q}}$ becomes a module algebra for $U_{q}^{r e s}$ : In the following we will use the restriction of this action to $U_{q}^{\text {res }}(\mathfrak{b}) \subset U_{q}^{\text {res }}$. As $U_{q}$ is not locally finite with respect to the adjoint action, this $U_{q}^{\text {res }}(\mathfrak{b})$-action does not integrate to a $B_{q}$-action. Thus $\mathcal{D}_{G_{q}}$ is not an object of $\mathcal{M}_{B_{q}}\left(G_{q}\right)$; however, $\mathcal{D}_{G_{q}}$ has a subalgebra $\mathcal{D}_{G_{q}}^{\text {fin }}=\mathcal{O}_{q} \star U_{q}^{\text {fin }}$ which belongs to $\mathcal{M}_{B_{q}}\left(G_{q}\right)$. This fact will be used below.

Definition 3.11. Let $\lambda \in T_{P}$. A $\left(B_{q}, \lambda\right)$-equivariant $\mathcal{D}_{G_{q}}$-module is a triple $(M, \alpha, \beta)$, where $M$ is a $\mathbb{C}$-module, $\alpha: \mathcal{D}_{G_{q}} \otimes M \rightarrow M$ a left $\mathcal{D}_{G_{q}}$-action and $\beta^{\text {res }}: M \rightarrow M \otimes \mathcal{O}_{q}(B)$ a right $\mathcal{O}_{q}(B)$-coaction. The latter action induces an $U_{q}^{\text {res }}(\mathfrak{b})$-action on $M$, again denoted by $\beta^{\text {res }}$. We have the natural map $U_{q}(\mathfrak{b}) \rightarrow$ $U_{q}^{\text {res }}(\mathfrak{b})$ which together with $\beta^{\text {res }}$ gives an action $\beta$ of $U_{q}(\mathfrak{b})$ on $M$. We require

i) The $U_{q}(\mathfrak{b})$-actions on $M \otimes \mathbb{C}_{\lambda}$ given by $\beta \otimes \lambda$ and by $\left(\left.\alpha\right|_{U_{q}(\mathfrak{b})}\right) \otimes$ Id coincide.

ii) The map $\alpha$ is $U_{q}^{\text {res }}(\mathfrak{b})$-linear with respect to the $\beta$-action on $M$ and the action on $\mathcal{D}_{G_{q}}$.

These objects form a category denoted by $\mathcal{D}_{B_{q}}^{\lambda}\left(G_{q}\right)$. There is the forgetful functor $\mathcal{D}_{B_{q}}^{\lambda}\left(G_{q}\right) \rightarrow \mathcal{M}_{B_{q}}\left(G_{q}\right)$. Morphisms in $\mathcal{D}_{B_{q}}^{\lambda}\left(G_{q}\right)$ are morphisms in $\mathcal{M}_{B_{q}}\left(G_{q}\right)$ that are $\mathcal{D}_{G_{q}}$-linear.

We defined $([\mathrm{BK}]) \mathcal{D}_{q}^{\lambda}$ as the maximal quotient of $\mathcal{D}_{G_{q}}$ which is an object of $\mathcal{D}_{B_{q}}^{\lambda}\left(G_{q}\right)$ and showed that

$$
\mathcal{D}_{q}^{\lambda}=\operatorname{Ind} M_{\lambda}
$$


as an object in $\mathcal{D}_{B_{q}}^{\lambda}\left(G_{q}\right)$. (See section 2.1.7 for the $B_{q}$-action $=U_{q}^{\text {res }}(\mathfrak{b})$-action on $M_{\lambda}$.) The global section functor $\Gamma: \mathcal{D}_{B_{q}}^{\lambda}\left(G_{q}\right) \rightarrow \mathcal{M}$ is the functor of taking $B_{q}$-invariants (with respect to the action $\beta$ ); we have $\Gamma=\operatorname{Hom}_{\mathcal{D}_{B_{q}}^{\lambda}\left(G_{q}\right)}\left(\mathcal{D}_{q}^{\lambda}\right.$, ).

Hence, in particular $\Gamma\left(\mathcal{D}_{q}^{\lambda}\right)=\operatorname{End}_{\mathcal{D}_{B_{q}}^{\lambda}\left(G_{q}\right)}\left(\mathcal{D}_{q}^{\lambda}\right)$ (which explains the ring structure on $\left.\Gamma\left(\mathcal{D}_{q}^{\lambda}\right)\right)$.

If we view $\mathcal{D}_{q}^{\lambda}$ as an object in ${ }_{G_{q}} \mathcal{M}_{B_{q}}\left(G_{q}\right)$, we have that $F r_{*}\left(\mathcal{D}_{q}^{\lambda}\right)$ is an object of $G_{q} \mathcal{M}(G / B)$. But it actually lies in ${ }_{G_{q}} \mathcal{M}_{B}\left(\mathcal{O} \otimes \mathcal{Z}_{-, \lambda}^{(l)}\right)$ since $M_{\lambda}$ is a $\mathcal{Z}_{-, \lambda}^{(l)}$-module. In the next section we shall show that $\mathcal{D}_{q}^{\lambda}=D$ and that the category of modules over this sheaf of algebras is $\mathcal{D}_{B_{q}}^{\lambda}\left(G_{q}\right)$.

3.3. $\mathcal{D}^{\lambda}$ as a sheaf of algebras. We have a natural functor:

$$
\begin{gathered}
F: \mathcal{D}_{B_{q}}^{\lambda}\left(G_{q}\right) \rightarrow \mathcal{M}_{B}\left(\mathcal{O} \otimes \mathcal{Z}_{-, \lambda}^{(l)}\right), \\
F(N)=N^{\mathfrak{b}_{q}} .
\end{gathered}
$$

Since taking $\mathfrak{b}_{q}$-invariants is exact on $B_{q}$-modules $\left(O_{q}(B)\right.$-comodules) (see (2.5) $)$ and is faithful on $\mathcal{M}_{B_{q}}\left(G_{q}\right)$ (see (2.4)), we get

Proposition 3.12. This functor is exact and faithful.

The functor $F$ has a left adjoint. Let us denote it $G$.

Proposition 3.13. $F\left(G\left(\mathcal{O} \otimes \mathcal{Z}_{-, \lambda}^{(l)}\right)\right)=\left(\mathcal{D}_{q}^{\lambda}\right)^{\mathfrak{b}_{q}}$ is a sheaf of algebras over $T^{\star} X^{\lambda}$. The category of modules over it is equivalent to $\mathcal{D}_{B_{q}}^{\lambda}\left(G_{q}\right)$.

Proof of Proposition 3.13. This theorem follows from a Barr-Beck type argument. $F$ is an exact and faithful functor with left adjoint $G$. $F \circ G$ is a monad in $q \operatorname{Coh}\left(T^{\star} X^{\lambda}\right)$ and modules over this monad are equivalent to the category $\mathcal{D}_{B_{q}}^{\lambda}\left(G_{q}\right)$. Note also that $F \circ G$ is a right exact functor from the category $q \operatorname{Coh}\left(T^{\star} X^{\lambda}\right)$ to itself. Note that any right exact functor $L$ from $q \operatorname{Coh}(X)$ to $q \operatorname{Coh}(Y)$ where $X$ and $Y$ are varieties is given by tensoring with the bimodule $L\left(O_{X}\right)$. In our case this is $F\left(G\left(\mathcal{O} \otimes \mathcal{Z}_{-, \lambda}^{(l)}\right)\right)$. The monad structure induces the algebra structure on this sheaf.

We want to prove the following:

Proposition 3.14. $\left(\mathcal{D}_{q}^{\lambda}\right)^{\mathfrak{b}_{q}}=D$ as sheaves of algebras.

Proof of Proposition 3.14, We have that

$$
\left(\mathcal{D}_{q}^{\lambda}\right)^{\mathfrak{b}_{q}}=\left(O_{q} \otimes M_{\lambda}\right)^{\mathfrak{b}_{q}}=\operatorname{Ind}_{\mathfrak{b}_{q}}^{G_{q}}\left(M_{\lambda}\right)=\operatorname{Ind}_{\mathfrak{u}_{q}}^{G_{q}} \circ \operatorname{Ind}_{\mathfrak{b}_{q}}^{\mathfrak{u}_{q}}\left(M_{\lambda}\right) .
$$

Hence it is enough to prove that

$$
\operatorname{Ind}_{\mathfrak{b}_{q}}^{\mathfrak{u}_{q}}\left(M_{\lambda}\right)=\operatorname{End}_{\mathcal{Z}_{-, \lambda}^{(l)}}\left(M_{\lambda+2 \rho}\right)
$$

and that this map is compatible with all relevant structures. Here we use two conventions about Verma modules, where $M_{\lambda}$ gets its action from the adjoint action of the restricted quantum group on the nonrestricted and in $\operatorname{End}_{\mathcal{Z}_{-, \lambda}^{(l)}}\left(M_{\lambda+2 \rho}\right)$ we think of the Verma module as induced from the Borel. It is enough to prove that

$$
\operatorname{Ind}_{\mathfrak{b}_{q}}^{\mathfrak{u}_{q}}\left(M_{\lambda, \chi}\right)=\operatorname{End}\left(M_{\lambda+2 \rho, \chi}\right) .
$$


First note that if we denote by $o_{q}(G)$ the functions on the quantum Frobenius kernel (the fiber over the identity of sheaf of algebras $O_{q}(G)$ over $O(G)$ ), we have that $o_{q}(G)=o_{q}\left(N_{-}\right) \otimes o_{q}(B)$ and so $\operatorname{Ind}_{\mathfrak{b}_{q}}^{\mathfrak{u}_{q}}\left(M_{\lambda, \chi}\right)=o_{q}\left(N_{-}\right) \otimes M_{\lambda, \chi}$ as vector spaces (actually as $N_{q}$-modules). Now by definition $M_{\lambda, \chi}$ is an algebra (it is a quotient of $\left.U_{q}(b)\right)$ and using duality, one can define an action of $o_{q}\left(N_{-}\right)$on $M_{\lambda, \chi}$ as in [J] (which gives explicit formulas on page 91 and proposition on page 92 ), getting the desired isomorphism by specializing the map from [J] (lemma on page 93) to the $\chi$ central character. Notice that [J] works with generic $q$ but that all the formulas and proofs make sense and are compatible with the action of the center for $q$ a root of unity if we work with the De Concini-Kac integral form.

3.3.1. Category $\mathcal{D}_{B_{q}}^{\lambda}\left(G_{q}\right)$ as a gluing of module categories over quantum Weyl algebras. In this section we will give another way of viewing $\mathcal{D}_{q}^{\lambda}$ as a sheaf of algebras over the cotangent bundle.

For $w \in \mathcal{W}$, De Concini and Lyubashenko DL introduced localizations $\mathcal{O}_{q, w}$ of $\mathcal{O}_{q}$. These induce localizations $\mathcal{O}_{w}$ of $\mathcal{O}$ corresponding to covering $G$ by translates of the big cell $B_{-} B$. Joseph has introduced localizations of the representation ring $\mathcal{O}_{q}^{N_{q}}([\mathrm{~J}])$, and it is easy to see that they are exactly $\mathcal{O}_{q, w}^{N_{q}}$. We thus have a covering of the category of $\mathcal{O}_{q}$-modules by the categories $\mathcal{O}_{q, w}$. By this we mean that we have a pair of adjoint functors

$$
f^{*}: \mathcal{O}_{q}-\bmod \rightarrow \prod \mathcal{O}_{q, w}-\bmod : f_{*}
$$

such that $f^{*}$ is exact and faithful, thus inducing an equivalence between $\mathcal{O}_{q}$-modules and modules over the monad $f^{*} \circ f_{*}$ (Barr-Beck). We will denote such an equivalence by

$$
\mathcal{O}_{q}-\bmod =\lim _{w \in \mathcal{W}} \mathcal{O}_{q, w}-\bmod
$$

We have corresponding localizations $\mathcal{M}_{B_{q}}\left(G_{q}\right)_{w}$ (i.e., $B_{q}$-equivariant $\mathcal{O}_{q, w^{-}}$ modules), of the category $\mathcal{M}_{B_{q}}\left(G_{q}\right)$. Then

$$
\mathcal{M}_{B_{q}}\left(G_{q}\right)=\lim _{w \in \mathcal{W}} \mathcal{M}_{B_{q}}\left(G_{q}\right)_{w} .
$$

Using the description of $\mathcal{M}_{B_{q}}\left(G_{q}\right)$ as a Proj-category from [BK], it is clear that $\mathcal{M}_{B_{q}}\left(G_{q}\right)_{w}$ is affine, i.e., $\mathcal{O}_{q, w}$ is a projective generator of $\mathcal{M}_{B_{q}}\left(G_{q}\right)_{w}$ and hence $\mathcal{M}_{B_{q}}\left(G_{q}\right)_{w} \cong \bmod -\operatorname{End}_{\mathcal{M}_{B_{q}}\left(G_{q}\right)}\left(\mathcal{O}_{q, w}\right)$. The functors that induce this equivalence are the adjoint pair $\left(? \otimes_{\operatorname{End}_{\mathcal{M}_{B_{q}}\left(G_{q}\right)}\left(\mathcal{O}_{q, w}\right)} \mathcal{O}_{q, w}, ?^{B_{q}}\right)$. Now $\operatorname{End}_{\mathcal{M}_{B_{q}}\left(G_{q}\right)}\left(\mathcal{O}_{q, w}\right)=$ $\operatorname{Hom}_{\mathcal{M}_{B_{q}}\left(G_{q}\right)}\left(\mathcal{O}_{q}, \mathcal{O}_{q, w}\right)=\Gamma\left(\mathcal{O}_{q, w}\right)$. Joseph [J] showed that

$$
\operatorname{End}_{\mathcal{M}_{B_{q}}\left(G_{q}\right)}\left(\mathcal{O}_{q, w}\right)=\left(M_{0}^{\star}\right)^{T_{w}}
$$

as $U_{q}$-modules, where $T_{w}$ are the algebra automorphisms of $U_{q}$ from section 2.1.3. Note that these algebras are nonisomorphic for different $w$ in general. When $w=e$, we have $\operatorname{End}_{\mathcal{M}_{B_{q}}\left(G_{q}\right)}\left(\mathcal{O}_{e}\right) \cong U_{q}\left(\mathfrak{n}_{-}\right)$as an algebra.

Similarly to $\mathcal{M}_{B_{q}}\left(G_{q}\right), \mathcal{M}_{B_{q}}\left(G_{q}\right)_{w}$ also has an induction functor from $B_{q}$-modules, $V \mapsto \mathcal{O}_{q, w} \otimes V$. It follows from the fact that $\mathcal{M}_{B_{q}}\left(G_{q}\right)_{w}$ is affine that

$$
\left(\mathcal{O}_{q, w} \otimes V\right)^{B_{q}} \cong \mathcal{O}_{q, w}^{B_{q}} \otimes V
$$


Next we have the forgetful functor $f_{\star}: \mathcal{D}_{B_{q}}^{\lambda}\left(G_{q}\right) \rightarrow \mathcal{M}_{B_{q}}\left(G_{q}\right)$, which is exact and faithful. It is easy to see that it has left adjoint $f^{\star}: \mathcal{M}_{B_{q}}\left(G_{q}\right) \rightarrow \mathcal{D}_{B_{q}}^{\lambda}\left(G_{q}\right)$ (just tensor with $\mathcal{D}_{G_{q}}$ over $\mathcal{O}_{q}$ and factor out the necessary relations).

Define $\mathcal{D}_{B_{q}}^{\lambda}\left(G_{q}\right)_{w}$ to be the localization of $\mathcal{D}_{B_{q}}^{\lambda}\left(G_{q}\right)$ lying over $\mathcal{M}_{B_{q}}\left(G_{q}\right)_{w}$ (i.e., replace $\mathcal{O}_{q}$ by $\mathcal{O}_{q, w}$ in the definition of $\mathcal{D}_{B_{q}}^{\lambda}\left(G_{q}\right)$ ). We get the adjoint pair of functors $\left(f_{w}^{\star}, f_{w, \star}\right)$ between these categories with the same properties as above. Hence, abstract nonsense shows that

Proposition 3.15. $f_{w}^{\star} \mathcal{O}_{q, w}=\mathcal{O}_{q, w} \otimes_{\mathcal{O}_{q}} \mathcal{D}_{q}^{\lambda}$ is a projective generator of $\mathcal{D}_{B_{q}}^{\lambda}\left(G_{q}\right)_{w}$ and therefore

$$
\mathcal{D}_{B_{q}}^{\lambda}\left(G_{q}\right)_{w} \cong \bmod -\operatorname{End}_{\mathcal{D}_{B_{q}}^{\lambda}\left(G_{q}\right)}\left(\mathcal{O}_{q, w} \otimes_{\mathcal{O}_{q}} \mathcal{D}_{q}^{\lambda}\right)
$$

Put

$$
\mathrm{A}_{q, w}^{\lambda}=\Gamma\left(\mathcal{O}_{q, w} \otimes_{\mathcal{O}_{q}} \mathcal{D}_{q}^{\lambda}\right)=\operatorname{End}_{\mathcal{D}_{B_{q}}^{\lambda}\left(G_{q}\right)}\left(\mathcal{O}_{q, w} \otimes_{\mathcal{O}_{q}} \mathcal{D}_{q}^{\lambda}\right)
$$

We can calculate these $\mathrm{A}_{q, w}^{\lambda}$ explicitly. We have

$$
\mathrm{A}_{q, w}^{\lambda}=\Gamma\left(\mathcal{O}_{q, w} \otimes_{\mathcal{O}_{q}} \mathcal{D}_{q}^{\lambda}\right)=\left(\mathcal{O}_{q, w} \otimes M_{\lambda}\right)^{B_{q}}=\mathcal{O}_{q, w}^{B_{q}} \otimes M_{\lambda}
$$

where the ring structure is induced from the one on $\mathcal{D}_{G_{q}}$.

It follows that the $\mathrm{A}_{q, w}^{\lambda}$ coincide with the rings introduced by Joseph [J]. In the generic case these can be described as the $B_{q}^{T_{w}}$-finite part of the full endomorphism ring $\operatorname{End}\left(\left(M_{\lambda}^{\star}\right)^{T_{w}}\right)$.

When $w=e$, Joseph describes this ring explicitly, a quantum Weyl algebra

$$
\mathrm{A}_{q, e}^{\lambda} \cong U_{q}\left(\mathfrak{n}_{-}\right) \otimes U_{q}\left(\mathfrak{n}_{+}\right) .
$$

Here, the algebra structures on $U_{q}\left(\mathfrak{n}_{-}\right)$and on $U_{q}\left(\mathfrak{n}_{+}\right)$are the usual ones and we have the commutation relations

$$
q^{-(\alpha, \beta)} E_{\alpha} \otimes F_{\beta}-q^{(\alpha, \beta)} F_{\beta} \otimes E_{\alpha}=\delta_{\alpha, \beta}
$$

for $\alpha, \beta \in \Delta$. (So $\mathrm{A}_{q, e}^{\lambda}$ 's algebra structure is independent of $\lambda$.)

The category $\mathcal{D}_{B_{q}}^{\lambda}\left(G_{q}\right)$ can now be described as the gluing of module categories

$$
\mathcal{D}_{B_{q}}^{\lambda}\left(G_{q}\right)=\lim _{w \in \mathcal{W}} \mathrm{A}_{q, w}^{\lambda}-\bmod .
$$

From the description of $\mathrm{A}_{q, w}^{\lambda}$ as $\mathcal{O}_{q, w}^{B_{q}} \otimes M_{\lambda}$ with the algebra structure induced from $\mathcal{D}_{G_{q}}$, we get

Proposition 3.16. i) $Z\left(\mathrm{~A}_{q, w}^{\lambda}\right)=\left(\mathcal{O}_{w} \otimes \mathcal{Z}_{-, \lambda}^{(l)}\right)^{B}=\mathcal{O}_{w}^{B} \otimes \mathcal{Z}_{-, \lambda}^{(l)}$ for each $w \in \mathcal{W}$.

ii) Consequently,

$$
q \operatorname{coh}\left(T^{\star} X^{\lambda}\right)=\lim _{w \in \mathcal{W}} Z\left(\mathrm{~A}_{q, w}^{\lambda}\right)-\bmod
$$

where $Z\left(\mathrm{~A}_{q, w}^{\lambda}\right)$ denotes the center of $\mathrm{A}_{q, w}^{\lambda}$.

Let us remark that for generic $\lambda$ the variety $\left(G \times N^{\lambda^{2 l}}\right) / B$ is an affine variety. 
3.3.2. Torsors. We first define a "torsor" as a category $\widetilde{\mathcal{D}}_{B_{q}}\left(G_{q}\right)$ of $\mathcal{D}_{G_{q}}$-modules that contains all $\mathcal{D}_{q}^{\lambda}, \lambda \in T_{P}$.

Definition 3.17. An object of $\widetilde{\mathcal{D}}_{B_{q}}\left(G_{q}\right)$ is a triple $(M, \alpha, \beta)$, where $\alpha: \mathcal{D}_{G_{q}} \otimes M \rightarrow$ $M$ is a left $\mathcal{D}_{G_{q}}$-action and $\beta^{\text {res }}: M \rightarrow M \otimes \mathcal{O}_{q}(B)$ is a right $\mathcal{O}_{q}(B)$-coaction.

i) The $U_{q}\left(\mathfrak{n}_{+}\right)$-actions on $M$ given by $\beta \mid U_{q}\left(\mathfrak{n}_{+}\right)$and by $\left(\left.\alpha\right|_{U_{q}\left(\mathfrak{n}_{+}\right)}\right)$coincide.

ii) The map $\alpha$ is $U_{q}(\mathfrak{b})$-linear with respect to the $\beta$-action on $M$ and the action on $\mathcal{D}_{G_{q}}$.

Put $\widetilde{\mathcal{M}}=U_{q}(\mathfrak{g}) / \sum_{\alpha \in R_{+}} U_{q}(\mathfrak{g}) \cdot E_{\alpha}$ (a "universal" Verma module) and define

$$
\widetilde{\mathcal{D}}_{\mathrm{q}}=\mathcal{O}_{q} \otimes \widetilde{\mathcal{M}}
$$

$\widetilde{\mathcal{D}}_{\mathrm{q}}$ inherits an $U_{q}^{\text {res }}(\mathfrak{b})$-module structure from $\mathcal{D}_{G_{q}}$, so $\widetilde{\mathcal{D}}_{\mathrm{q}}$ is an object in $\widetilde{\mathcal{D}}_{B_{q}}\left(G_{q}\right)$.

$\widetilde{\mathcal{M}}$ has the $U_{q}^{r e s}(\mathfrak{b})$-submodule $\mathcal{Z}_{\mathfrak{b}}^{(l)}=\mathcal{Z}^{(l)} /\left(\mathcal{Z}^{(l)} \cap U_{q} \cdot U_{q}\left(\mathfrak{n}_{+}\right)_{+}\right)$, which is an algebra generated by $K_{\mu}^{(l)}, E_{\mu}^{(l)}, \mu \in R_{+}$. $b_{q}$ acts trivially on $\mathcal{Z}_{\mathfrak{b}_{-}}^{(l)}$, so the $U_{q}^{\text {res }}(\mathfrak{b})$ action factors to a $U(\mathfrak{b})$-action on $\mathcal{Z}_{\mathfrak{b}-}^{(l)}$. We have

Lemma 3.18. As a $B$-module, $\mathcal{Z}_{\mathfrak{b}_{-}}^{(l)} \cong \mathcal{O}(B)$, where the module structure comes as follows: consider the map $B \rightarrow B$ defined as $t n \rightarrow t^{2} n$; this is an unramified covering. Use this covering to pull back the adjoint action of $B$ on itself .

Proof of Lemma 3.18. To calculate this, one should notice that in the generic case the action induced from the adjoint action of $U_{q}\left(n_{+}\right)$on the universal Verma module is the same as the one induced from the commutator action. Hence one can use the calculations of $\mathrm{CKP}$ which give the required result. As for the torus part, the adjoint action is by the grading and it is easy to see that we get the required action.

As in the previous sections we see that $\mathcal{O}(G \times B)$ imbeds to $\widetilde{\mathcal{D}}_{\mathrm{q}}, B_{q}$-equivariantly. Note that this embedding corresponds to the surjection ()$^{l}: T_{P} \rightarrow T$. Define $B^{\text {twist }}$ to be the cover of $B$ induced from the map $B=T N \ni t n \rightarrow t^{2 l} n \in B$ with the natural $B$-structure. We have

Definition-Proposition 3.19. We define $\widetilde{\mathrm{T}}^{\star} \mathrm{X}^{\text {twist }}=\left(G \times B^{\text {twist }}\right) / B$ (where $B$

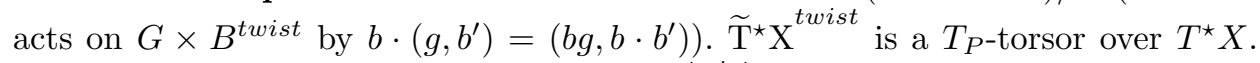
$\widetilde{\mathcal{D}}_{\mathrm{q}}$ is a sheaf of Azumaya algebras over $\widetilde{\mathrm{T}}^{\star} \mathrm{X}^{\text {twist }}$.

3.4. Azumaya splitting over fibers. Recall the $U_{\mathcal{A}}^{r e s}$-action on $U_{q}$. The center of $U_{q}$ is exactly the submodule of invariants with respect to the subalgebra $u_{q}$. Hence the center of $U_{q}$ is a $\mathfrak{g}$-module. Recall also that $U^{\text {fin }}$ is the finite part of $U_{q}$ with respect to the $U_{\mathcal{A}}^{r e s}$-action. We have

Lemma 3.20. Let $\mathcal{Z}^{\text {fin }}$ be the center of $U^{\text {fin }}$. We have $\mathcal{Z}^{\text {fin }}=\mathcal{Z} \cap U^{\text {fin }}$. $Z^{\text {fin }}$ is an integrable $\mathfrak{g}$-module and $\operatorname{Spec}\left(\mathcal{Z}^{\text {fin }}\right)$ is isomorphic to an unramified cover of $G \times_{T / W} T$ where the map $T \rightarrow T / W$ is induced from the l'th power map and the action is the adjoint action.

Proof of Lemma 3.20. The first claim follows from Joseph's JL] description of $U_{q}^{\text {fin }}$ inside $U_{q}$. The second statement follows from the calculations of [CKP]. 
Consider the diagram

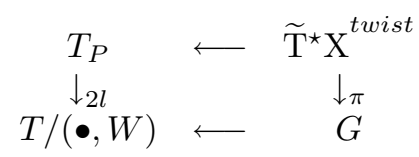

Definition 3.21. $t \in T_{P}$ is unramified if for every $\alpha \in \tilde{\Delta}=\Delta \cup\left\{-\alpha_{0}\right\}$ (where $\alpha_{0}$ is the longest root), $\left(t\left(K_{\alpha}\right)\right)^{2 l}=1$ implies $\left(t\left(K_{\alpha}\right)\right)^{2}=q^{-(2 \rho, \alpha)} . T_{P}^{u n r a m}$ denotes the set of unramified $t$ 's.

Note that any $t \in T_{P}$ can be made unramified by adding an integral weight to it. We put $\mathcal{Z}^{\text {unram }}=\mathcal{O}\left(T_{P}^{\text {unram }} \times_{T / W} B_{-} B\right)$ and $U_{q}^{\text {unram }}=U_{q} \otimes_{\mathcal{Z}^{H C}} \mathcal{O}\left(T_{P}^{\text {unram }}\right)$. Brown and Gordon $\mathrm{BG}$ proved that

Lemma 3.22. i) $U_{q}^{\text {unram }}$ is Azumaya over $\mathcal{Z}^{\text {unram }}$ and

ii) for each $\chi \in \operatorname{maxspec}\left(\mathcal{Z}^{\text {unram }}\right)$, we have $\operatorname{End}\left(M_{\chi}^{\text {baby }}\right)=U_{q} / \chi U_{q}$ (where $M_{\chi}^{b a b y}$ is the baby Verma module).

Let $\sigma: \widetilde{\mathrm{T}}^{\star} \mathrm{X}^{\text {twist }} \rightarrow T_{P} \times_{T / W} G$ be the natural map. Note that this is a $G_{q^{-}}$ equivariant map. Now since both sheaves are also $G_{q}$-equivariant, from the description of the $\mathcal{D}_{G_{q}}$ as induced from endomorphism of baby Vermas and from Lemma 3.22 that describes the sheaf over a dense subset (the big cell), we have

Proposition 3.23. The action map $U_{q}^{\text {fin }} \otimes_{Z} O_{\widetilde{\mathrm{T}}^{\star} \mathrm{X}^{t w i s t}} \rightarrow \widetilde{\mathcal{D}}_{\mathrm{q}}$ induces an isomorphism $\left.U_{q}^{\text {fin }} \otimes_{Z} O_{\widetilde{\mathrm{T}} \star \mathrm{X}^{t w i s t}, \text { unram }} \cong \widetilde{\mathcal{D}}_{\mathrm{q}}\right|_{\widetilde{\mathrm{T}}^{\star} \mathrm{X}^{\text {twist } \text {,unram }}}$.

Thus we see that over the preimage of the big cell $\mathcal{D}_{q}^{\lambda}$ is Azumaya. (Note again that in a formal neighbourhood of $p$ it is Azumaya everywhere.)

Hence it follows as in BMR (vanishing of the Brauer group of a local ring with separably closed residue field) that $\widetilde{\mathcal{D}}_{\mathrm{q}}$ Azumaya splits over the formal neighborhood of any fiber of $\sigma$ lying over the big cell. Hence, we get

Corollary 3.24. The category of $\mathcal{D}_{q}^{\lambda}$-modules supported on the fiber of $\sigma$ over the big cell is equivalent to $\mathcal{O}$-modules supported on the same fiber.

\subsection{Derived $\mathcal{D}$-affininty.}

3.5.1. Global sections and vanishing cohomology of $\mathcal{D}_{q}^{\lambda}$.

Proposition 3.25. We have i) $U_{q}^{\lambda} \cong R \Gamma\left(\mathcal{D}_{q}^{\lambda}\right)$ and ii) $\widetilde{U}_{q} \cong R \Gamma\left(\widetilde{\mathcal{D}}_{\mathrm{q}}\right)$ (if l is a prime $p>$ Coxeter number of $G$ ).

We recall from $\left[\mathrm{BK}\right.$ that the natural map $U_{q}^{\lambda} \rightarrow \Gamma\left(\mathcal{D}_{q}^{\lambda}\right)$ was given as follows: There is the natural surjection $U_{q}^{\lambda} \rightarrow M_{\lambda}$. It induces a surjective map

$$
\text { Ind } U_{q}^{\lambda} \rightarrow \operatorname{Ind} M_{\lambda}=\mathcal{D}_{q}^{\lambda} \text {. }
$$

Since $U_{q}^{\lambda}$ is a $G_{q}$-module, (2.7) shows that $\Gamma\left(\operatorname{Ind} U_{q}^{\lambda}\right)=U_{q}^{\lambda}$, which gives the desired map, by applying $\Gamma$ to (3.27).

Remark 3.26. Recall that $U_{q}^{\lambda}=U^{f i n} / J_{\lambda}$. In order to get global differential operators equal to $U_{q} / J_{\lambda}$, one can enlarge $\mathcal{D}_{q}^{\lambda}$ by adding some grading operators; the vanishing of higher self-extensions will remain true. 
Proof of Proposition 3.25. We have $\mathcal{D}_{q}^{\lambda}=\operatorname{Ind} M_{\lambda}$. We have the integral version Ind $M_{\lambda, \mathcal{A}} \in \mathcal{M}_{B_{q}}\left(G_{q}\right)_{\mathcal{A}}$.

Consider the specialization $\mathcal{A} \rightarrow \mathbb{F}_{p}, \nu \rightarrow 1$. In [BK] we showed that the statement about global sections in $i$ ) holds for a generic $q$. The argument given there transforms to the case of $p$ 'th roots of unity if we can show that

$$
\operatorname{dim}_{\mathbb{C}} \Gamma\left(\operatorname{Ind~gr}_{i} M_{\lambda}\right)=\operatorname{dim}_{\mathbb{C}} \Gamma\left(\operatorname{Ind} \operatorname{gr}_{i} M_{\lambda, q=1}\right) .
$$

Now, by [AJ], $\operatorname{dim}_{\mathbb{C}} \Gamma\left(\operatorname{Ind} \operatorname{gr}_{i} M_{\lambda, q=1}\right)=\operatorname{dim}_{\mathbb{F}_{p}} \Gamma\left(\operatorname{Ind} \operatorname{gr}_{i} M_{\lambda, \mathbb{F}_{p}}\right)$. On the other hand, it follows from $\mathrm{APW}$ that $\operatorname{dim}_{\mathbb{C}} \Gamma\left(\operatorname{Ind~gr}_{i} M_{\lambda}\right)=\operatorname{dim}_{\mathbb{F}_{p}} \Gamma\left(\operatorname{Ind~gr}_{i} M_{\lambda, \mathbb{F}_{p}}\right)$.

In order to prove that higher cohomologies vanishes in $i$ ), it suffices by APW] (page 26) to show that

$$
R \Gamma_{\mathbb{F}_{p}}^{>0}\left(\text { Ind } M_{\lambda, \mathbb{F}_{p}}\right)=0 .
$$

This holds because $R \Gamma^{>0}\left(\operatorname{Ind} \operatorname{gr} M_{\lambda, \mathbb{F}_{p}}\right)=R \Gamma^{>0}\left(\operatorname{Ind} S\left(\mathfrak{n}_{-, p}\right)\right)=0$, by [A.] (gr is taken with respect to the filtration on $M_{\lambda}$ coming from the identification $M_{\lambda}=$ $U_{q}\left(\mathfrak{n}_{-}\right)$and putting each $F_{\mu}$ in degree 1$)$. This proves $i$ ). The proof of $i i$ ) is similar.

Remark. In $\mathrm{BK}$ we explain how the restrictions on the roots of unity can be dropped by using the work of $[\mathrm{K}]$ or $[\mathrm{W}]$ in order to drop some of the assumptions of APW.

3.5.2. Localization functor. Recall the definition of the localization functor $L o c_{\lambda}$ : $U_{q}^{\lambda}-\bmod \rightarrow \mathcal{D}_{B_{q}}^{\lambda}\left(G_{q}\right)$ [BK].

Definition 3.27. Define the localization functor $\operatorname{Loc}_{\lambda}: \Gamma\left(\mathcal{D}_{q}^{\lambda}\right)-\bmod \rightarrow \mathcal{D}_{B_{q}}^{\lambda}\left(G_{q}\right)$ by $M \rightarrow \mathcal{D}_{q}^{\lambda} \otimes_{U_{q}^{\lambda}} M$, where we have used $U_{q}^{\lambda}=\Gamma\left(\mathcal{D}_{q}^{\lambda}\right)$.

This is a left adjoint to the global sections functor. Note that $\operatorname{Loc}_{\lambda}\left(U_{q}^{\lambda}\right)=$ $\mathcal{D}_{q}^{\lambda}$. Similarly, we can define localization $\widetilde{L o c}: U_{q}^{f i n}-\bmod \rightarrow \widetilde{\mathcal{D}}_{B_{q}}\left(G_{q}\right)$ and also localizations with generalized central character $\widetilde{\operatorname{Loc}} \lambda: \bmod ^{\lambda}\left(U_{q}^{f i n}\right) \rightarrow \widetilde{\mathcal{D}}_{B_{q}}\left(G_{q}\right)^{\lambda}$. Since $U_{q}^{\text {fin }}$ has finite cohomological dimension, we get that $\widetilde{\operatorname{Loc}}$ has left derived functor

$$
\widetilde{\mathcal{L}}: D^{b}\left(U_{q}^{f i n}-\bmod \right) \rightarrow D^{b}\left(\widetilde{\mathcal{D}}_{B_{q}}\left(G_{q}\right)\right)
$$

and hence also $\widetilde{\operatorname{Loc}}^{\lambda}$. Note that a priori we do not know if $U_{q}^{\lambda}$ has finite cohomological dimension (it will follow later that for regular $\lambda$ it has); hence $L o c_{\lambda}$ has a left derived functor which is defined for the bounded above derived category:

$$
\mathcal{L}_{\lambda}: D^{-}\left(U_{q}^{\lambda}-\bmod \right) \rightarrow D^{-}\left(\mathcal{D}_{B_{q}}^{\lambda}\left(G_{q}\right)\right) .
$$

For regular $\lambda$ this functor is compatible with restriction from a generalized central character as in BMR and so we get that it is also defined on the bounded derived category. From now on we will use $\mathcal{L}_{\lambda}$ to denote the functor on the bounded derived category in case $\lambda$ is regular. We thus have

Lemma 3.28. (i) $\widetilde{\mathcal{L}}$ is left adjoint to $R \widetilde{\Gamma}$ and (ii) if $\lambda$ is regular, $\mathcal{L}_{\lambda}$ is left adjoint to $R \Gamma$.

If $\lambda$ is not regular, the second statement in the lemma will hold if we replace $D^{b}$ by $D^{-}$. 
Proposition 3.29. $i)$ The functor $R \widetilde{\Gamma} \circ \widetilde{\mathcal{L}}: D^{b}\left(U_{q}\right) \rightarrow D^{b}\left(\widetilde{U}_{q}\right)$ is isomorphic to the functor $M \rightarrow M \otimes_{\mathcal{Z}^{H C}} \mathcal{O}\left(T_{P}\right)$.

ii) For regular $\lambda$, the adjunction map $i d \rightarrow R \Gamma \circ \mathcal{L}_{\lambda}$ is an isomorphism.

Proof. Part $i$ ) follows from part ii) of Lemma 3.25 for free modules and then from the same lemma again for general modules, by considering free resolutions.

For $i$ ) observe that for regular $\lambda$, for any $M \in D^{b}\left(U_{q}^{\lambda}-\bmod \right)$, we have canonically $M \otimes_{\mathcal{Z}^{H C}} \mathcal{O}\left(T_{P}\right)=\bigoplus_{w \in \mathcal{W}} \bigoplus_{\mathrm{Hom}(P,\{+1,-1\})} M$. Now the claim follows since $R \Gamma \circ$ $\mathcal{L}_{\lambda}(M)$ is one of these direct summands.

3.5.3. Derived $\mathcal{D}$-affinity.

Theorem 3.30. $R \Gamma: D^{b}\left(\mathcal{D}_{B_{q}}^{\lambda}\left(G_{q}\right)\right) \rightarrow D^{b}\left(U_{q}^{\lambda}-\bmod \right)$ is an equivalence of categories.

Noting that the canonical bundle of $T^{\star} X^{\lambda}$ is trivial and that $\pi$ from (3.26) is a projective morphism, the theorem now follows from the following lemma of [BMR]:

Lemma 3.31. Let $\mathcal{A}$ be a generically Azumaya algebra over a smooth variety $X$ (i.e., Azumaya over a generic point). Suppose that $X$ is Calabi-Yau (i.e., $\omega_{X} \cong$ $\left.\mathcal{O}_{X}\right)$ and that we have a projective map $\pi: X \rightarrow$ SpecR for some commutative algebra $R$. Suppose also that the derived global section functor $R \Gamma: D^{b}(\mathcal{A}-\bmod ) \rightarrow$ $D^{b}(\Gamma(\mathcal{A})-\bmod )$ has a right adjoint $\mathcal{L}$ and the adjunction morphism id $\rightarrow R \Gamma \circ \mathcal{L}$ is an isomorphism. Then $R \Gamma$ is an equivalence of categories.

The first point in the proof of this lemma is the following proposition:

Proposition 3.32. Let $L: C \rightarrow D$ be a functor between triangulated categories with $D O$-triangulated Calabi-Yau for some finitely generated commutative algebra $O$ (we have a natural functor $R \operatorname{Hom}(X, Y)$ defined on $D \times D^{o p}$ with values in $D^{b}(O-$ mod) whose first cohomology is the Hom in the category and there is a functorial isomorphism $R \operatorname{Hom}(X, Y) \cong \mathbb{D}_{O}(R \operatorname{Hom}(Y, X[n]))$ where $\mathbb{D}$ is the duality functor over $O)$. Suppose $L$ has a right adjoint $R$ such that the adjunction $i d \rightarrow R \circ L$ is an isomorphism. Suppose also that $D$ is indecomposable. Then $L$ is an equivalence.

The second point is the observation that the bounded derived category of modules over an Azumaya algebra is indecomposable.

Note that, for generic $\lambda, T^{\star} X^{\lambda}$ is affine and hence we then get an equivalence $\Gamma: \mathcal{D}_{B_{q}}^{\lambda}\left(G_{q}\right) \cong U_{q}^{\lambda}-\bmod$.

\section{Applications}

Assume for simplicity $\lambda$ is integral and regular and that $\chi$ belongs to $B_{-} B$ and is unipotent. We know that

$$
D^{b}\left(\mathcal{D}_{B_{q}}^{\lambda}\left(G_{q}\right)\right) \cong D_{\lambda}^{b}\left(U_{q}-\bmod \right) .
$$

We get that

$$
D^{b}\left(\mathcal{D}_{B_{q}}^{\lambda}\left(G_{q}\right)_{(\chi, \lambda)}\right) \cong D_{\hat{\chi}, \lambda}^{b}\left(U_{q}-\bmod \right)
$$

where the left hand side denotes those (complexes of) $\mathcal{D}_{q}^{\lambda}$-modules supported on the Springer fiber of $(\chi, \lambda)$ and the right hand side denotes $U_{q}^{\lambda}$-modules that are locally annihilated by a power of the maximal ideal in $\mathcal{Z}$ corresponding to $\chi$ (generalized central $l$-character $\chi$ ). 
By the Azumaya splitting we have

$$
\mathcal{D}_{B_{q}}^{\lambda}\left(G_{q}\right)_{(\chi, \lambda)} \cong q \operatorname{coh}\left(T^{\star} X\right)_{\chi}
$$

where the latter category is the quasi-coherent $\mathcal{O}$-modules on $T^{\star} X$ supported on the Springer fiber of $\chi$ (with respect to the usual Springer resolution $T^{\star} X \rightarrow \mathcal{N}=$ unipotent variety of $G$ ). Note that for the trivial central character this equivalence is Koszul dual to the equivalence in $\mathrm{ABG}$, thus giving a geometric proof of their equivalence.

We deduce an equivalence of $K$-groups

$$
K_{\hat{\chi}}\left(U_{q}^{\lambda}-\bmod \right) \cong K\left(q \operatorname{coh}\left(T^{\star} X\right)_{\chi}\right) .
$$

Note that $K\left(q \operatorname{coh}\left(T^{\star} X\right)_{(\chi)}\right)$ is torsion-free. From (4.4) we see for instance that the

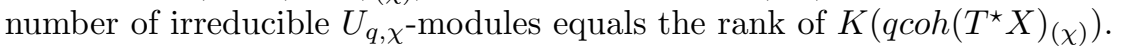

In $[\mathrm{Kr}$ we use the methods here to prove a conjecture made by De Concini, Kac and Processi stating that the dimensions of irreducible $U_{q, \chi}$-modules is divisible by $l^{\operatorname{codim}(O) / 2}$ where $l$ is the order of the root of unity and $O$ is the orbit through $\chi$.

Notice also that [BMR] showed that $K\left(q \operatorname{coh}\left(T^{\star} X\right)_{\chi}\right) \cong K\left(q \operatorname{coh}\left(T^{\star} X\right)_{\chi}\left(\overline{\mathbb{F}}_{p}\right)\right)$ for $p>$ Coxeter number of $G$. This relates our work to the representation theory of $\mathfrak{g}_{p}$ via the results in [BMR].

Actually, it is possible to relate our work to $\mathrm{BMR}$ in order to get more transparent geometric proofs of the theorems appearing in the work of Andersen, Jantzen and Soergel [AJS, leading to the proof of Lusztig's conjecture about multiplicities in characteristic $p$ and also extending them to nontrivial central characters. Our work defines a perverse $t$-structure on $O$-modules in zero characteristic (root of unity), where [BMR] defines such a structure in positive characteristic. By showing that in specializing the root of unity $t$-structure one would get (at least for big $p$ ) the positive characteristic $t$-structure, one would be able to deduce that the quantum modules and the positive characteristic modules have the same multiplicity formulas, as shown in AJS. This will appear in future work.

\section{ACKNOWLEDGEMENTS}

We thank Joseph Bernstein and David Kazhdan for many useful conversations.

\section{REFERENCES}

[ABG] S. Arkhipov, R. Bezrukavnikov, V. Ginzburg, Quantum groups, the loop Grassmannian, and the Springer resolution, J. Amer. Math. Soc. 17 (2004), 595-678. MR2053952 (2005g:16055)

[AG] S. Arkhipov, D. Gaitsgory, Another realization of the category of modules over the small quantum group, Adv. Math. 173 (2003), no. 1, 114-143. MR.1954457 (2004e:17010)

[AJ] H. H. Andersen, J. Jantzen, Cohomology of induced representations for algebraic groups, Math. Ann. 269 (1984), 487-525. MR766011 (86g:20057)

[AJS] H. H. Andersen, J. Jantzen, W. Soergel, Representations of quantum groups at a pth root of unity and of semisimple groups in characteristic p: independence of $p$, Asterisque 220 (1994). MR 1272539 (95j:20036)

[APW] H. H. Andersen, P. Polo and Wen Kexin, Representations of quantum algebras, Invent. Math. 104 (1991), 1-59. MR1094046 (92e:17011)

[BK] E. Backelin and K. Kremnizer, Quantum flag varieties, equivariant quantum $\mathcal{D}$-modules, and localization of Quantum groups, Adv. in Math. 203 (2006), 408-429. MR2227727 (2007b:17021)

[BB] A. Beilinson and J. Bernstein, Localisation de $\mathfrak{g}$-modules, C. R. Acad. Sc. Paris 292 (Série I) (1981), 15-18. MR610137 (82k:14015) 
[BMR] A. Bezrukavnikov, I. Mirkovic and D. Rumynin, Localization for a semi-simple Lie algebra in prime characteristic, arXiv:math.RT/0205144.

[BG] K. A. Brown, I. Gordon, The ramification of centres: Lie algebras in positive characteristic and quantized enveloping algebras, Math. Z. 238 (2001), 733-779, arXiv:math.RT/9911234. MR1872572 (2002i:17027)

[CP] W. Chari and A. Pressley, A guide to quantum groups, Cambridge University Press, Cambridge 53 (1995). MR1358358 (96h:17014)

[CKP] C. De Concini, V.G. Kac and C. Procesi, Quantum coadjoint action, JAMS v.5, number 1 (1992), 151-189. MR1124981 (93f:17020)

[DL] C. De Concini and V. Lyubashenko, Quantum function algebras at roots of 1 , Adv. in Math. 108 (1994), 205-262. MR1296515 (95m:17014)

[J] A. Joseph, Faithfully flat embeddings for minimal primitive quotients of quantized enveloping algebras, In A. Joseph and S. Shnider (eds.), Quantum deformation of algebras and their representations, Israel Math. Conf. Proc. 7 (1993), pp. 79-106. MR1261902 (94m:17013)

[JL] A. Joseph, G. Letzter, Local finiteness for the adjoint action for quantized enveloping algebras, J. Algebra 153 (1992), 289-318. MR.1198203 (94b:17023)

[K] M. Kaneda, Cohomology of infinitesimal quantum algebras, Journal of Algebra 226 (2000), 250-282. MR1749888 (2001a:20084)

[Kr] K. Kremnizer Proof of the De Concini-Kac-Processi conjecture, math/0611236.

[W] D. Woodcock, Schur algebras and global bases: New proofs of old vanishing theorems, Journal of Algebra 191 (1997), 331-370. MR.1444503 (98d:17027)

Departamento de Matemáticas, Universidad de Los Andes, Carrera 4, 26-51, Bogota, Colombia

E-mail address: erbackel@uniandes.edu.co

Department of Mathematics, Massachusetts Institute of Technology, 77 MassaChusetts Avenue, Cambridge, Massachusetts 02139-4307

E-mail address: kremnize@math.mit.edu 